Thomas Gil

Bedingungen und Einstellungen 

Bedingungen und Einstellungen Thomas Gil 



\title{
Bedingungen und Einstellungen
}

\author{
Thomas Gil
}

Logos Verlag Berlin

$\lambda$ oros 


\section{(9) (1) $(\Theta$}

Bibliografische Information der Deutschen Nationalbibliothek

Die Deutsche Nationalbibliothek verzeichnet diese Publikation in der Deutschen Nationalbibliografie; detaillierte bibliografische Daten sind im Internet über http://dnb.d-nb.de abrufbar.

(C) Copyright Logos Verlag Berlin GmbH 2021

Alle Rechte vorbehalten.

ISBN 978-3-8325-5296-1

Logos Verlag Berlin GmbH

Georg-Knorr-Str. 4, Geb. 10

12681 Berlin

Tel.: $\quad+49(0) 30 / 42851090$

Fax: $\quad+49(0) 30 / 42851092$

https://www.logos-verlag.de 


\section{Inhaltsverzeichnis}

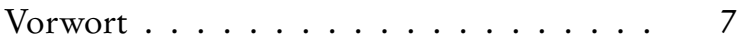

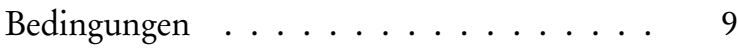

Einstellungen ................ 19

Literaturverzeichnis . . . . . . . . . . . . . 27

Anhang: Realismus und Realismuskritik . . . 29 



\section{Vorwort}

Um Bedingungen und Einstellungen geht es in den folgenden Essays. Natürliche, ökonomische und soziokulturelle Bedingungen bestimmen und markieren unser Leben. Wir werden ohne unsere willentliche Zustimmung geboren und ihnen ausgesetzt. Erst im Laufe unseres Lebens lernen wir, mit ihnen mehr oder weniger angemessen umzugehen und im Rahmen unserer Möglichkeiten einige von ihnen zu verändern.

Lebend entwickeln wir Einstellungen in Bezug auf die Welt: epistemische, optativische, emotionale, evaluative und intentionale bzw. prospektive Einstellungen. Diese Einstellungen machen unser geistiges Leben aus, das aus einer Reihe von mentalen Zuständen, Ereignissen und Funktionen besteht.

Um uns als reale Wesen geht es also: um uns als geistige Wesen in der Welt.

Im Anhang (unter dem Titel „Realismus und Realismuskritik“) wird die reflektierte „realistische“ Einstellung, die den vorgetragenen Überlegungen zugrunde liegt, explizit Thema. 



\section{Bedingungen}

Die einzelnen Menschen kommen immer zu spät. Sie werden geboren und in eine Welt gesetzt, die (ontologisch betrachtet) bereits möbliert ist. In ihr gibt es spezifisch beschaffene Einzeldinge und Dinge, die in den meisten Fällen nach bestimmten Gesetzmäßigkeiten geschehen. Natürlichen Sprachen werden sie ausgesetzt, die wohlgeordnete Sprachgebilde kennen und für ein komplexes Denken unentbehrlich sind. Die einzelnen Menschen kommen zu spät. In einem nicht immer schnell durchschaubaren Weltgeschehen müssen sie lernen, sich zu orientieren. Und das korrekte Sprechen und das angemessene Denken lernen sie im Zuge vieler Interaktionen mit anderen Menschen und in Gruppen sich konform verhaltender Individuen. Ihr Leben wird deswegen ein bestimmtes, bedingtes Leben sein, dessen Bedingungen ihnen gegeben wurden. Wohl werden sie irgendwann hier und da bestimmend und bedingend handeln können. Die Ausgangslage ist für alle klar und eindeutig: Bedingungen bestimmen das Leben von Menschen. Bedingungen machen aber auch ein solches Leben allererst möglich.

Um Bedingungen geht es im Folgenden: um Bedingungen, die in Konditionalsätzen festgehalten werden, regelförmig sein können, der Beschaffenheit und den Funktionsweisen existierender Dinge 
entstammen, Handlungs- und Lebenssituationen ermöglichen und mit denen die Individuen mehr oder weniger gut, mehr oder weniger angemessen umzugehen lernen.

\section{Konditionalsätze und Regularitäten}

In unseren natürlichen Sprachen gibt es (grammatiktheoretisch ausgedrückt) „Konditionalgefüge“, die Kombinationen aus einem „Bedingungssatz“ (in einem eigentlichen Sinne) und seinem zugehörigen „Hauptsatz“ sind. In solchen Konditionalgefügen drückt der Vordersatz eine Voraussetzung für die Geltung des weiteren Satzes aus. Oder: In Konditionalgefügen wird etwas ausgedrückt, das nur der Fall ist oder sein wird, wenn eine bestimmte Bedingung erfüllt ist. „Wenn A, B“ ist die logische Form einer solchen Konditionalkonstruktion.

Es gibt zwei Arten von relevanten Konditionalkonstruktionen resp. Konditionalsätzen: indikativische und konjunktivische. Diese Differenz scheint wichtig zu sein, denn die Wahrheitsbedingungen für indikativische Konditionalsätze sind andere als die Wahrheitsbedingungen für konjunktivische Bedingungssätze. Dieser Differenz versucht David Lewis mit seiner Vorstellung „möglicher Welten“ gerecht zu werden. 
Epistemologisch orientierte Autoren sind daran interessiert zu analysieren, wie Bedingungssätze im menschlichen Leben funktionieren. Sie rücken das hypothetische Denken und Argumentieren in den Mittelpunkt ihrer Untersuchungen, um zu markieren, wie vielfältig die Formen von „Konditionalisierung" im Umgang mit und in der Verarbeitung von Informationen sind. Die für Konditionalkonstruktionen übliche Formel von Antecedenssatz („protasis“) und Konsequenssatz (,apodosis“) wird im hypothetischen Denken nicht immer verwendet. Häufig geschieht die Konditionalisierung auf indirekte oder versteckte Weise. Außerdem kann die Konditionalisierung nicht nur in assertorischen Sätzen geschehen, sondern auch in Frage-, Befehls-, Versprechens- und Empfehlungssätzen. Konditionalsätze sind allgegenwärtig im Denken menschlicher Individuen.

Vielfältig sind die Verwendungen von Konditionalsätzen sowie die Funktionen, die sie in der Lage sind zu vollziehen, wie Nicholas Rescher in seinem Buch „Conditionals" anschaulich vorführt. Konditionalsätze können nämlich dazu dienen: Mutmaßungen vorzutragen; Phänomene (kausal) zu erklären; Prozesse zu beschreiben; Absichten und Vorhaben zu äußern; auf Gewohnheiten und Dispositionen aufmerksam zu machen; Versprechen, Entschuldigungen, Drohungen auszudrücken; Hand- 
lungsanweisungen zu geben; Restriktionen festzuhalten; Verhandlungen zu gestalten (Rescher, 13f.).

Rein grammatikalisch lässt das „Wenn“ der Formel „Wenn A, B“ eine Vielfalt von Variationen zu: „selbst wenn“, „nur wenn“, „wenn nur“, „wenn und nur wenn", „wie wenn“, ,vorausgesetzt, dass“ oder „es sei denn“ (Rescher, 8ff.).

Im Folgenden steht allerdings nicht das hypothetische Denken im Mittelpunkt, das mit Voraussetzungen und Annahmen arbeitet und Konditionalsätze verwendet, sondern die grundsätzliche Konditionalität, die unser Sein, Denken und Handeln wesentlich bestimmt.

Im Natürlichen, im Denken und beim Handeln und Entscheiden sind wir mit Bedingungen konfrontiert, die wir selbst nicht geschaffen haben, mit denen wir aber rechnen müssen.

Einen Gedankengang Erwin Schrödingers aus seiner Antrittsrede an der Universität Zürich mit dem Titel „Was ist ein Naturgesetz?“ variierend, könnte man formulieren: Die Erfahrung zeigt, dass natürliche Vorgänge nicht mit kaleidoskopischer Willkür einander folgen, sondern dass in ihnen erhebliche Regelmäßigkeit zutage tritt und dass die theoretische Erfassung einer solchen Regelmäßigkeit für die Lebenspraxis sehr nützlich sein kann. 
Allerdings übernimmt jeder, der über die Erfahrung hinausgehende „absolute Naturgesetze“ (E. Schrödinger) postuliert, die den (nicht-statistischen) Charakter von Kantischen „synthetischen Prinzipien a priori“ hätten, eine schwer zu tragende Beweislast. So, oder ähnlich zu reden, ist keine Kapitulation des Denkens vor der Wirklichkeit, sondern der Versuch, ein mögliches Missverständnis dessen, was Naturgesetze nur sein können, zu vermeiden.

Es ist nicht unklug, wenn es um Gesetzmäßigkeiten geht, sich an die Argumentation David Humes anzuschließen. Für den Anfang. Wir beobachten, wie Hume klar darstellt, wie Phänomene oder Vorkommnisse anderen Phänomenen und Vorkommnissen folgen, also: ein Nacheinander von Geschehnissen und Ereignissen, und auf dieser Basis unterstellen wir, dass es kausale Verknüpfungen gibt. Durch die Beobachtung von Regularitäten, die sich wiederholen, kommen wir demnach zu der Vorstellung der Kausalität und des Bewirkens von etwas durch etwas anderes.

Wenn wir aber erklären wollen, warum ein solches Nacheinander von Vorkommnissen zustande kommt, scheint das Feststellen einer Regularität nicht genug zu sein. Epistemologisch macht es immer Sinn, nicht zu viel zu behaupten. Deswegen ist Humes Sichtweise eine sehr gute Weise des Denkens und des Räsonierens. Für den Anfang. Eine Frage 
stellt sich, die rein „humeanisch“ nicht zufriedenstellend zu beantworten ist. Wie kommt es dazu, dass beispielsweise die Einnahme eines Medikamentes (d.h. eines bestimmten chemischen Stoffes) da$\mathrm{zu}$ führt, dass ein bestimmter Schmerz aufhört? Das bloße Insistieren auf einer nicht-kausalen Regularität erweist sich nun als nicht ausreichend. Vernünftig ist an dieser Stelle der Hinweis darauf, dass das Medikament bestimmte Eigenschaften oder Dispositionen (dispositionale Qualitäten) hat, die tatsächlich bewirken, dass etwas passiert, das ohne die Einnahme des Medikamentes nicht passiert wäre. Selbstverständlich ist eine solche hinweisende Behauptung grundsätzlich fallibel. Aber in den meisten Fällen irren wir nicht, wenn wir solche Behauptungen machen. Es ist in der Tat sinnvoll, davon auszugehen, dass tatsächlich die existierenden Dinge, die die Welt möblieren, eine bestimmte Beschaffenheit haben, die dafür sorgt, dass in gewissen Situationen Einiges, das sich beobachten lässt, geschieht.

Kräfte, Dispositionen, Situationen

Im „Phaidros“ schildert Platon, was der gute Dialektiker tut. Er erarbeitet erstens präzise Definitionen. Wie der gute Metzger, „schnitzt“ er zweitens an den „Gelenken der Wirklichkeit“. Dieser Bestim- 
mung Platons kann man entnehmen, dass die unabhängig von uns existierende Wirklichkeit Artikulationen, Gelenke, ja eine Struktur hat, und zwar wie das zu zerschneidende Tier für den Metzger. Wie ist diese Formulierung Platons, die u.a. von David Lewis aufgegriffen wurde, genau zu verstehen? Eine mögliche Interpretation wäre folgende. Es gibt Veränderungen, Variationen und Ko-Variationen im Wirklichen, die auf der Basis der objektiven Beschaffenheit der involvierten Dinge zustande kommen.

Die objektive Beschaffenheit der Dinge erweist sich im Einzelnen als eine Gesamtheit von (charakterisierenden und dispositionalen) Merkmalen, Eigenschaften und Qualitäten. Insbesondere die sogenannten dispositionalen Eigenschaften (die Dispositionen) sind wichtig, wenn es darum geht, was geschieht, d.h. das Vorkommen von etwas, die stattfindenden Veränderungen und Transformationen zu erklären. Dispositionen sind Eigenschaften von Dingen, so Mumford und Molnar u.a., die in bestimmten Situationen oder Konstellationen dadurch manifest werden, dass sie etwas bewirken. Wenn die Dinge, die die Wirklichkeit ausmachen, eine Beschaffenheit haben, so ist eine solche Beschaffenheit für uns etwas, das wir in unserem Denken und Handeln konsequent in Rechnung stellen müssen.

„Situationen“ sind strukturelle Zusammenhänge, in denen Einzeldinge (auch Individuen), Relatio- 
nen und Raum-Zeit-Gebiete so zusammenkommen, dass etwas geschieht.

In unseren natürlichen Sprachen verwenden wir Sätze, die sich auf Situationen beziehen. Eine Analyse solcher Sätze ermöglicht uns, die Komponenten zu erfassen, die eine bestimmte Situation ausmachen. Sich auf Situationen beziehende Sätze geben in der Regel an, was geschieht, wann, wo und wie es geschieht.

In Situationen geschieht immer, was geschieht: in einer bestimmten Konstellation, an einem bestimmten Ort, zu einer bestimmten Zeit und mit einer Beteiligung von bestimmten Faktoren, Größen und Entitäten.

In Situationen werden auch die Dispositionen und Vermögen der existierenden Dinge manifest, indem sie etwas bewirken oder am Geschehenden in einer spezifischen Weise beteiligt sind. Nur in Situationen sind Bedingungen in ihrer bestimmenden Macht erfahrbar. Und nur in Situationen entstehen Möglichkeiten und Spielräume des Handelns.

Objektive Verhältnisse und Bedingungen bestimmen das individuelle und das kollektive Leben von menschlichen Individuen und Gruppen. Dies heißt aber keineswegs, dass es für Individuen und Gruppen keine Gestaltungsmöglichkeiten gibt. Im Gegenteil. Nur im Bedingten und Bestimmten kann es 
Selbstbestimmung, Autonomie geben. Mit anderen Worten: Objektive Bedingungen verschlingen nicht Selbstbestimmungs- und Freiheitsräume. Im Bestimmten, Bedingten gibt es immer Möglichkeiten, die nur konkret und in der jeweiligen Situation auszuloten sind. Die Geschichte der menschlichen Gattung belegt, dass Kreativität, Innovationen, wissenschaftliche und künstlerische Schöpfungen von ihren Urhebern den beschränkten Verhältnissen geradezu abgerungen worden sind.

Wenn Bedingungen und Bestimmungen allgegenwärtig sind, dann kann die in der neuzeitlichen Philosophie so zentral gewordene "Selbstbestimmung“ nur die responsive Kompetenz des situationsangemessenen Sich-Bestimmen-Lassens sein. Was dies „ex ante“, „ex post“ und „in actu“ genau heißen könnte, hat Martin Seel versucht, vorsichtig, tentativ zu beschreiben (Seel, 288f.). 



\section{Einstellungen}

Wenn im Folgenden die Rede von „Einstellungen“ ist, so sind nicht die "Einstellungen“ gemeint, die Psychologinnen und Psychologen erforschen. Psychologisch bestimmt, ist eine "Einstellung“ eine Bereitschaft von Individuen, in bestimmter Weise auf Personen, Gruppen, Objekte, Situationen oder Vorstellungen (wertend) zu reagieren, eine Reaktionsbereitschaft also, die sich in Annahmen oder Überzeugungen, Gefühlen und Neigungen, oder aber im Verhalten ausdrücken kann. In der Regel sind die Einstellungen, die Psychologen untersuchen, erfahrungsbasiert. Und die psychologische Forschung konzentriert sich häufig auf die Thematik der Einstellungsänderung. Solche Einstellungen können Einstellungen der Ablehnung von, der Neutralität gegenüber oder der Akzeptanz von „x“ sein, wobei „x“ für (wie bereits gesagt) Personen, Gruppen, Objekte, Situationen oder Vorstellungen stehen kann.

Der Begriff der Einstellungen soll hier in einem weiten Sinne verwendet werden, und zwar für alle jene mentalen Zustände und Prozesse, die das konkrete geistige Leben von Individuen kennzeichnen. Er referiert also auf Überzeugungen, Annahmen, Wünsche, Gefühle und Emotionen, Bewertungen und Stellungnahmen sowie Absichten und 
Pläne. Mit anderen Worten: all jene Größen und Phänomene, die den Geist, d.h. die Praxis des Geistigen, ausmachen.

Nur auf drei geistige Phänomene habe ich meine Aufmerksamkeit konzentriert: auf das, was wir glauben, d.h. die Überzeugungen, auf das, was wir wollen und wünschen, die Wünsche, sowie auf das, was wir vorhaben und beabsichtigen, die Absichten.

\section{Überzeugungen, Wünsche, Absichten}

Will man Menschen, die belebte "geistige" Wesen sind, verstehen, so ist das Phänomen des "Glaubens“ bzw. des „Überzeugungen Habens“ oder des „Überzeugt-Seins von etwas“ unverzichtbar. Die „beseelten“ Lebewesen, die Menschen nach Aristoteles sind, haben Überzeugungen, d.h. glauben, dass p. Dies ist auch die Struktur der im menschlichen Leben so basalen Glaubenssätze: „x glaubt, dass p“, „x glaubt, dass irgendetwas der Fall ist“ oder „X glaubt, dass irgendetwas wahr ist".

Im Austausch mit ihrer Umwelt machen Menschen Erfahrungen. Sie erhalten Informationen, die sie speichern und verarbeiten. Auf der Basis ihrer Erlebnisse und Erfahrungen bilden sie Annahmen, Vorstellungen und Überzeugungen über die Welt, über das, was es gibt, über andere Menschen, über sich selbst. Ihre weiteren Erfahrungen werden sol- 
che Annahmen und Überzeugungen bestätigen oder aber widerlegen.

Solche Annahmen und Überzeugungen erfüllen viele Funktionen. Sie machen Vorhersagen möglich. Sie ermöglichen das verstehende Erklären von Sachverhalten. Sie rufen gefühlsmäßige bzw. emotionale Haltungen hervor. Sie ermöglichen Handlungen und Entscheidungen. Sie vermitteln Zuversicht und Vertrauen.

Annahmen und Überzeugungen gibt es nie einzeln. Sie haben wir immer in Netzwerken oder Systemen von Überzeugungen, in denen sie sich gegenseitig stützen und verstärken.

Als geistiges Phänomen ist das Haben von Überzeugungen, d.h. das Glauben, ein eigenständiges, nichtreduzierbares Phänomen. „Glauben“ ist außerdem ein Zustand, in dem sich ein betreffendes Subjekt über eine gewisse (sei es auch beliebig kurze) Zeit hinweg befindet. Ein Zustand und kein Ereignis. Glauben ist ein intentionales, auf etwas bezogenes Phänomen. Es hat deswegen einen (propositionalen) Gehalt. Man glaubt etwas oder man glaubt, dass etwas der Fall (bzw. wahr) ist.

Man kann etwas glauben, ohne sich dessen bewusst $\mathrm{zu}$ sein. Allerdings ist es so, dass, wer etwas glaubt, gewöhnlich in der Lage ist, sich seines Glaubens bewusst zu werden. Es liegt nämlich im Begriff des 
Glaubens, dass einer, der dieses oder jenes glaubt, sich auch dessen bewusst ist, dass er es glaubt.

Unser Begriff von Glauben lässt sich nicht auf andere zurückführen, die uns gleichermaßen vertraut und unabweisbar wären.

Als geistiges Wesen kann man nicht nur etwas glauben. Man kann auch etwas wünschen. Man kann x wünschen, oder man kann wünschen, dass $\mathrm{p}$ (dass irgendetwas geschieht oder der Fall ist). Immer wenn man wünscht, ist man intentional auf etwas bezogen: einen Gegenstand, ein Ereignis, einen Sachverhalt.

„Optativische“ Sätze sind Sätze, die Wünsche ausdrücken. „Optativische“ Einstellungen sind die Einstellungen, die Wünsche sind. Bei optativischen Einstellungen gibt es verschiedene Komponenten, die im jeweiligen Fall mehr oder weniger ausgeprägt sein können. Eine Person, die etwas wünscht, hat im Regelfall zum Gewünschten eine positive Einstellung, die in der Literatur häufig als „evaluative" Komponente genannt wird. Dazu kommt die wichtige dispositionale oder "motivationale“ Komponente, die dazu führt, dass etwas getan wird, um den Wunsch zu verwirklichen. Bei der tatsächlichen Verwirklichung des Wunsches kommt es zu einer Befriedigung oder Lustempfindung, die man die „satisfaktive“ Komponente nennen könnte. 
Häufig werden die Wünsche mit bestimmten Handlungen oder Verhaltensweisen identifiziert, und dies in der Absicht, nicht zu viele (mentale) Entitäten oder Größen zu postulieren. Die Frage danach, ob es neben einem bestimmten Tun, einer bestimmten Handlung oder einem bestimmten Verhalten, die der Wunsch wären, doch nicht etwas Anderes gibt, das eine solche „sparsame“ Konzeption nicht berücksichtigen würde, ist ganz berechtigt. Denn in der Tat gibt es Wünsche, die sich nicht im Handeln manifestieren und dennoch existent sein können. Wir kennen alle viele Fälle von einem bloßen Wünschen ohne Handlungen, von einem Wünschen also, das sich nicht im realen Geschehen manifestiert. In Bezug auf das Wollen hat Gottfried Seebass sieben Phänomene von „verrichtungsunabhängigen“ oder verrichtungsseparaten Willensereignissen unterschieden: u.a. bloßes Wollen, Überlegungen zur Realisierbarkeit des Gewollten, spätere Verwirklichungen usf. (Seebass, 165). Ähnliches ließe sich vom Wünschen behaupten. Wenn dies sich so verhält, wäre die Frage zu stellen, was es sein könnte, das zusätzlich zum Handeln oder zum Verhalten käme, wenn irgendjemand etwas wünscht. Die Beantwortung dieser Frage wäre schwierig und sicher nicht in einzelfallunabhängiger Weise zu leisten. 
„Wünschen“ ist ein komplexer mentaler, geistiger Vorgang. Definitive Analysen gibt es nicht. Wohl aber gibt es viele sinnvolle Überlegungen und Unterscheidungen, die erhellend sind. Neben der wichtigen von H. Frankfurt gemachten Unterscheidung von Wünschen „erster Ordnung (die auf Objekte bezogen sind) und Wünschen „zweiter Ordnung“ (die auf andere Wünsche bezogen wären) scheint mir jene Unterscheidung sinnvoll zu sein, die auf die Differenz von primären und abgeleiteten Wünschen (Peter Stemmer redet von ,intrinsischen“ und „extrinsischen“ Wünschen, S. 231) aufmerksam macht.

Von der allgemeinen „Intentionalität“ geistiger Einstellungen, die alle Überzeugungen, Wünsche und Präferenzen charakterisiert, müsste man die einzelnen „Intentionen“ bzw. „Absichten“, die einzelne Handelnde bilden und verfolgen, unterscheiden. Diese sind Vorhaben und Projekte, die die Handelnden $\mathrm{zu}$ verwirklichen versuchen. In vielen Fällen ist es sinnvoll, um unnötige Verdinglichungen zu vermeiden, das Beabsichtigen adverbial oder adjektivisch auszudrücken und statt von „Absichten“ von "absichtlichen" Spaziergängen, ,absichtlichen“ Einkäufen oder „absichtlichen“ Aktivitäten zu sprechen. Eine solche Versprachlichung zeigt, dass der introspektive Weg zur Erforschung von Absichten, wie Elisabeth Anscombe betont, „nutzlos“ ist (Anscombe, 149). 
„Absichten“ sind Momente von „Geschichten“ und nur in solchen ganzen Zusammenhängen markieren sie relevante Differenzen. Manchmal kann man zwar einzelne „Absichten“ profilieren, die dominierend sind. Doch häufig sind solche Absichten keine absoluten „atomistischen“ Einheiten, sondern Teile von Plänen und Strategien, worauf u.a. Michael E. Bratman immer wieder insistiert. „Absichten“ wären demnach in den meisten Fällen in „webs of regularities and norms" eingebettet (Bratman, 1987, 10). Bratmans funktionalistisches sogenanntes „Package-Deal“-Modell wendet sich gegen die zu simple Auffassung, welche Absichten als isolierte mentale Größen denkt, die man separat untersuchen könnte. Auf einen Punkt gebracht, heißt es bei Bratman: „intentions are ... the building blocks of ... plans; and plans are intentions writ large" (Bratman, 1987, 8). 



\section{Literaturverzeichnis}

Anscombe, G. E. M., Absicht, Freiburg i. Br. 1986. Barwise, J., Perry, J., Situationen und Einstellungen. Grundlagen der Situationssemantik, Berlin 1987.

Bratman, M. E., Intention, Plans, and Practical Reason, Cambridge, Massachusetts 1987.

Bratman, M. E., Faces of Intention. Selected Essays on Intention and Agency, Cambridge 1999.

Gergen, K. J., Gergen, M. M., Social Psychology, New York 1986.

Gil, T., Conflicts, Machines, Beliefs, and Decisions, Berlin 2019.

Hirsch, E., Dividing Reality, Oxford 1997.

Jackson, F. (Hrsg.), Conditionals, Oxford 1991.

Kemmerling, A., Glauben. Essay über einen Begriff, Frankfurt a. M. 2017.

Kusser, A., Dimensionen der Kritik von Wünschen, Frankfurt a. M. 1989.

Lewis, D., Philosophical Papers, Band II, Oxford 1986.

Molnar, G., Powers. A Study in Metaphysics, Oxford 2009.

Mumford, L., Dispositions, Oxford 1998. 
Platon, Phaidros oder Vom Schönen, Stuttgart 2016.

Rescher, N., Conditionals, Cambridge, Massachusetts 2007.

Schrödinger, E., Was ist ein Naturgesetz? Beiträge zum naturwissenschaftlichen Weltbild, München 1962.

Seebass, G., Wollen, Frankfurt a. M. 1993.

Seel, M., Sich bestimmen lassen. Studien zur theoretischen und praktischen Philosophie, Frankfurt a. M. 2002.

Stemmer, P., Der Vorrang des Wollens. Eine Studie zur Anthropologie, Frankfurt a. M. 2016.

Williamson, T., Suppose and Tell. The Semantics and Heuristics of Conditionals, Oxford 2020.

Zimbardo, P. G., Psychologie, Berlin 1992. 


\section{Anhang: Realismus und Realismuskritik}

Im Alltag sind wir alle Realisten: „robuste“ Realisten. Nicht weil wir über eine Theorie des Realismus verfügten, sondern weil wir eine realistische Einstellung eingenommen haben, die sich in der Vergangenheit bewährt hat und sich immer wieder in der Gegenwart bewährt.

Wenn es regnet, sagen wir nicht, dass wir Regenempfindungen haben oder, wie „Empiriokritizisten" sagen würden, dass wir Regenempfindungskomplexe haben. Wir sagen einfach, dass es regnet.

Kritisch betrachtet bzw. wohl überlegt und in den Einzelwissenschaften, in denen es Theorien gibt, sind wir aber nicht durchgängig Realisten. Bereichsspezifisch sind wir manchmal Realisten und manchmal Nichtrealisten oder Antirealisten. So können wir beispielsweise im Bereich der Mathematik die Ansicht vertreten, dass die mathematischen Gegenstände nicht unabhängig von uns existieren, und im Bereich der Moral der Meinung sein, dass es keine "moralischen Tatsachen“ gibt.

Es ist sinnvoll deswegen drei verschiedene Arten von Realismus zu unterscheiden: den Alltagsrealismus, einen wohlüberlegten oder reflektierten Realismus und den theoretischen Realismus. 
Der Alltagsrealismus ist keine Theorie. Er ist weder eine axiomatisierte Theorie mit Axiomen, Definitionen, Postulaten und Theoremen noch eine Theorie im Sinne eines Systems von Beobachtungsätzen und theoretischen Sätzen. Er ist auch keine Menge von mathematischen Strukturen, Gleichungen oder formalen Notationen. Und er ist auch nicht ein explorativer Forschungsprozess, in dem Begriffe gebildet und Theorierahmen entwickelt werden. Der robuste Alltagsrealismus ist eine „Einstellung“.

Die alltagsrealistische Einstellung ist durch zwei Einsichten charakterisiert. Diese Einsichten werden nicht als Überzeugungen oder Thesen vertreten und verteidigt. Vielmehr sind sie Teil einer Praxis: sie sind im Alltagshandeln inkorporiert und realisiert. Die erste Einsicht wäre die „Existenzeinsicht“. Sie geht davon aus, dass es Dinge, Sachverhalte, Entitäten und Objekte gibt. Die zweite Einsicht ist die „Unabhängigkeitseinsicht“. Sie geht davon aus, dass solche existierenden Objekte unabhängig von unserem Denken, unabhängig von unserem Erkennen existieren, dass sie geistunabhängig sind, dass der Geist sie nicht konstruiert, schafft, synthetisiert, stiftet oder konstituiert.

Ich habe von „Einsichten“ gesprochen. José Ortega y Gasset hätte von „creencias“ („Glaubensgewissheiten“) und nicht von „Ideen“ oder „Meinungen“ (,ideas“) gesprochen. Für Ortega y Gasset erwer- 
ben wir Ideen und Meinungen. Für sie argumentieren wir und wir versuchen manchmal, sie zu begründen oder zu rechtfertigen. „Glaubensgewissheiten“ hätten wir nicht, wir seien sie.

Ludwig Wittgenstein sprach von "Gewissheiten“ und er war der Meinungen, dass es keinen Sinn mache, solche „Gewissheiten“ in Frage zu stellen. Denn so würden wir halt denken und handeln. Nach Wittgenstein würde es auch keinen Sinn machen, das Verb „wissen“ in einem Zusammenhang mit „Gewissheiten“ zu verwenden, denn wir würden von den Gewissheiten als Selbstverständlichkeiten ausgehen: sie wären selbstverständliche Annahmen oder Prämissen unseres Denkens und Handelns, und nicht Überzeugungen oder Meinungen, die wir vertreten und die wahr oder falsch sein könnten.

Man könnte behaupten, dass eine überlegte, reflektierte realistische Einstellung (nicht die Einstellung des Alltagsrealismus) in der Regel „lokal“ ist, d.h. bereichsspezifisch und nicht "global“. Die meisten reflektierten oder theoretischen Realisten sind Realisten in bestimmten Bereichen und in Bezug auf bestimmte Entitäten (und nicht in allen Bereichen und in Bezug auf alle Entitäten). Akzeptierte man diese Begrifflichkeit, die drei Arten von Realismus unterscheidet, so wären „globale theoretische Antirealisten" viele Idealisten, kritische Idealisten und Kantianer sowie Verifikationisten. 
In der sogenannten „Realismus-Antirealismus“-Debatte (seit den 70er Jahren des vergangenen Jahrhunderts) gibt es eine Reihe von Alternativen zum theoretischen Realismus. Die Alternativen oder Optionen zum theoretischen Realismus, die viele Varianten und Unterarten kennen, wären: der Perspektivismus, der Emotivismus in der Moraltheorie, die verschiedenen Konstruktivismen (auch der Kantische oder kritizistische Konstruktivismus), der Fiktionalismus, die diversen Spielarten eines Interpretationismus, der Verifikationismus sowie der Instrumentalismus.

Die kritischen Argumente gegen den theoretischen (oder semitheoretischen) Realismus entstammen auch diesen Formen und Gestalten eines theoretischen Antirealismus. Solche kritischen Argumente sind ganz ernst zu nehmen. Sie reichen aber nicht aus, um einen "globalen" theoretischen Antirealismus zu begründen. Nun zu einigen dieser kritischen Argumente und den Bereichen, in denen sie vorgetragen worden sind. Ich werde mich auf den Bereich der Mathematik, den Bereich der einzelnen Wissenschaften und den Bereich der Moral konzentrieren.

Man könnte im Bereich der Mathematik davon ausgehen, dass die mathematischen Objekte (Zahlen, Gleichungen und formale Notationen) nicht real existieren (etwa in einem platonischen Ideenhimmel), sondern dass sie Begriffsapparatur sind, mit 
der wir arbeiten und die sich hervorragend bewährt hat. Man würde dann eine nicht-realistische, nichtplatonistische Position vertreten, vielleicht eine „formalistische“ oder eine „intuitionistische“ bzw. „konstruktivistische“ Position.

Es wäre allerdings unangemessen, eine solche nichtrealistische Position zu generalisieren, d.h. vom Mathematikbereich ausgehend zu behaupten, dass der Realismus bzw. realistische Positionen überhaupt nicht verteidigt werden können oder überhaupt nicht $\mathrm{zu}$ vertreten sind.

Sicher, „verifikationistische“ Positionen machen viel Sinn in Bezug auf mathematische Sätze. Aber dies hängt mit der ganz besonderen Rolle zusammen, die Verifikationen (d.h. Beweise und Widerlegungen) in der Mathematik spielen. Für einen "globalen Verifikationismus" reichen sie allerdings nicht aus.

In den einzelnen Wissenschaften hat man es mit verschiedenen Arten von Objekten zu tun: mit observablen Gegenständen und mit theoretischen Größen und Entitäten, die man abduktiv einführt, um bestimmte Phänomene zu erklären. Viele Wissenschaftlerinnen sind der Meinung, dass die theoretischen Terme nicht referieren, wie die Beobachtungsterme dies tun. Sie wären, so heißt es weiter, „Mittel“, die man unterstellend einsetzt, lediglich „Instrumente“. So argumentierend kommen sie zu 
einer „instrumentalistischen“ Kritik realistischer Positionen.

Für den „Instrumentalismus“ in der Wissenschaftsphilosophie geht es um empirische Adäquatheit, nicht um Wahrheit. Empirische Adäquatheit besagt nicht unabhängige Existenz der postulierten oder gesetzten theoretischen Größen. Die Frage, um die es dabei geht, wäre: Gibt es oder gibt es nicht „Klassen“, „Schichten“, Stände“, „Elektronen“, „Teilchen“, „Atome“, „Streuungen“, „Strahlungen"?

Nur eine einzelfallbezogene Diskussion kann hier meines Erachtens weiterhelfen. Sie würde zeigen, dass es einige Beweislasten für fiktionalistische und instrumentalistische Positionen tatsächlich gibt.

Leichter zu entkräften sind „perspektivistische“ Argumentationen, die auf die Standortgebundenheit von Beobachtungen aufmerksam machen. Sie sind ernst zu nehmen. Sie sind berechtigt, aber auch trivial. Aus ihnen lässt sich keine globale antirealistische Position ableiten.

Im Bereich der Moral sind die sogenannten „Emotivisten" der Meinung, dass das moralische Sollen nichts Anderes als der Ausdruck der subjektiven Präferenzen von einzelnen Individuen oder Gruppen ist. „Emotivismus“, „Subjektivismus“ und „Relativismus" liegen in der Moraltheorie nahe beiein- 
ander, ja sie sind Bündnispartner. Sie koinzidieren darin, dass sie behaupten, „moralische Tatsachen“ gäbe es nicht.

Es ist nicht erklärungsbedürftig, dass es in einer Welt ohne Menschen keine „moralischen Tatsachen" geben würde. Aber wo es Menschen gibt, die bestimmte berechtigte Interessen haben, gibt es objektiv Gutes, das diese Menschen vielleicht nicht immer faktisch wollen oder anstreben, das sie aber, kritisch betrachtet und wohl überlegt, wollen und anstreben sollten. Es kann wirklich vorkommen, dass ich „" “ oder "y“ tatsächlich nicht will. Aber es ist wohl möglich, dass ich, kritisch geprüft, „x“ oder "y“ wollen sollte, so dass " $\mathrm{x}^{\text {“ und } ~} \mathrm{y}$ “ unabhängig von meinen faktischen Präferenzen für mich gelten, ja moralisch gelten, weil sie objektiv wünschenswert sind.

Beim Denken und beim Handeln sind wir mit einer Reihe von Bedingungen konfrontiert, die unabhängig von uns existieren. Sie müssen wir in Rechnung stellen, wenn wir angemessen denken und handeln wollen. Diese Bedingungen hängen mit der Beschaffenheit und mit den „dispositionalen“ Eigenschaften der Dinge, die unsere Umwelt ausmachen, zusammen. Sie hängen weiter mit Gesetzmäßigkeiten und Regularitäten im Weltgeschehen zusammen. 
In „Konditionalsätzen“ beziehen wir uns auf solche Bedingungen. „Wenn A, dann B“ (oder „wenn A, $B$ “) ist die logische Form von Konditionalaussagen. Das so ausgedrückte „Konditionalgefüge“ besagt, dass etwas nur der Fall ist oder sein wird, wenn eine bestimmte Bedingung erfüllt ist. Eine besondere Unterart von Konditionalaussagen bzw. von sprachlichen Konditionalkonstruktionen sind die sogenannten „kontrafaktischen Konditionalaussagen“ der Form: „Wenn A der Fall wäre, dann B“ bzw. „wenn A nicht der Fall wäre, dann B“.

Diese Konditionalaussagen und die kontrafaktischen Konditionalsätze standen hier nicht im Mittelpunkt. Thema war die Konditionalität, die in solchen Konditionalkonstruktionen ausgedrückt wird. Und diese Konditionalität gibt es nur, weil es unterschiedliche Bedingungsverhältnisse in der Welt gibt, die wir nicht geschaffen haben, die wir aber mehr oder weniger gut begreifen und sprachlich darstellen können. Diese Bedingungsverhältnisse sind „objektiv" in dem Sinne, dass sie unabhängig von uns existieren. Auf sie nehmen wir sprachlich-begrifflich Bezug und sie müssen wir berücksichtigen, wenn wir in unserem Denken und Handeln erfolgreich sein wollen. 

Natürliche, ökonomische und soziokulturelle Bedingungen bestimmen das Leben menschlicher Individuen und Gruppen, die verschiedene Einstellungen gegenüber dem, was es gibt, entwickeln können und immer wieder lernen, mit dem Bestehenden auf unterschiedliche Weise umzugehen.

\title{
Logos Verlag Berlin
}

\section{ISBN 978-3-8325-5296-1}

\author{
ISSN 2567-1758
}

